\section{Impact of Implementing a Pediatric Early Warning System (PEWS) in a Pediatric Oncology Hospital}

\author{
Asya Agulnik, $M D, M P H^{*}+$; \\ Sherry Johnson, MSN, RN, CPON, NE-BCł; \\ Regina Wilkes, MSN, RN, CNL, CCRN‡; \\ Lane Faughnan, RN*; Angela Carrillo, PhD*; \\ R. Ray Morrison, $M D \dagger$
}

before and after PEWS implementation). Unplanned PICU transfers arriving via RRT increased from $11.5 \%$ to $60.5 \%$ of admissions $(P<0.0001$, Fig. 3).

Conclusions/Implications: PEWS can be successfully implemented in a pediatric hematology-oncology/HSCT hospital with improvement in accuracy over time. Implementation of PEWS resulted in increased use of the RRT system and more patients with clinical deterioration arriving to the PICU via an organized escalation pathway, representing standardization of care and improvement in the culture of safety in the hospital.

Key words: Pediatric Early Warning System, Pediatric Oncology, PEWS, Rapid Response Team, Cancer, Hematopoietic Stem Cell Transplant, Clinical Deterioration

Background: Hospitalized pediatric oncology and hematopoietic stem cell transplant (HSCT) patients have frequent clinical deterioration requiring transfer to the Pediatric Intensive Care Unit (PICU). Pediatric Early Warning Systems (PEWS) can aid early identification of clinical deterioration and improve outcomes in these patients.

Objectives: Describe the impact of PEWS implementation in a dedicated pediatric hematology-oncology/HSCT hospital. Methods: A PEWS tool and escalation algorithm were implemented between August and October 2016 (Fig. 1). Implementation quality was evaluated by measuring errors in PEWS calculation, omissions, and algorithm activation. Frequency of emergency activations and method of unplanned PICU transfer were compared before and after PEWS implementation.

Results: Random monitoring of PEWS scores demonstrated $12.7 \%$ calculation errors, 3.8\% omissions, and 1.7\% algorithm errors. Omissions and algorithm errors decreased over time following implementation ( $P<0.0001$ and $P=0.005$, respectively). Frequency of Rapid Response Team (RRT) activations increased after PEWS implementation from 1.3 to 12.2/1,000-inpatient-days $(P<0.0001)$, without an increase in Code Blue activations (1.2 to $0.97 / 1,000$-inpatient-days, $P=0.49$, Fig. 2) and no change in frequency of unplanned PICU transfers (5.7/1,000-inpatient-days
From the *Department of Global Pediatric Medicine, St. Jude Children's Research Hospital, Memphis, Tenn.; †Division of Critical Care, Department of Pediatrics, St. Jude Children's Research Hospital, Memphis, Tenn.; and $¥$ Nursing Administration, St. Jude Children's Research Hospital, Memphis, Tenn.

- ${ }^{*}$ Corresponding author. Address: Asya Agulnik, MD, MPH, 262 Danny Thomas Place, Mail Stop 721, Memphis, TN 38105. Email: asya.agulnik@stjude.org PH: 901-595-0367; FAX: 901-595-3122.

- Statement of Authorship: All authors meet the journal's authorship requirements.

Ethical Adherence: This study was given a quality improvement (nonresearch) designation by the St. Jude Children's Research Hospital IRB.

Copyright (C) 2018 the Author(s). Published by Wolters Kluwer Health, Inc. This is an open-access article distributed under the terms of the Creative Commons Attribution-Non Commercial-No Derivatives License 4.0 (CC$B Y-N C-N D)$, where it is permissible to download and share the work provided it is properly cited. The work cannot be changed in any way or used commercially without permission from the journal.

To Cite: Agulnik A, Johnson S, Wilkes R, Faughnan L, Carrillo A, Morrison RR. Impact of Implementing a Pediatric Early Warning System in a Pediatric Oncology Hospital. Pediatr Qual Saf 2018;3:065.

Received for publication February 8, 2018; Accepted February 12, 2018.

Published online April 17, 2018.

DOI: 10.1097/pq9.0000000000000065

\section{REFERENCE}

1. Agulnik A, Forbes PW, Stenquist N, et al. Validation of a Pediatric Early Warning Score in hospitalized pediatric oncology and hematopoietic stem cell transplant patients. Pediatr Crit Care Med. 2016;17:e146-e153. 
A

\section{St. Jude Advanced Warning Score}

\begin{tabular}{|c|c|c|c|c|c|}
\hline Behavior/Neuro & 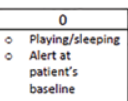 & 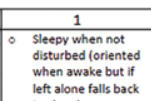 & 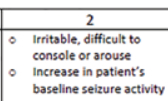 & 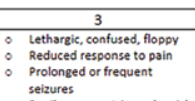 & Score \\
\hline Cardiovasecular & 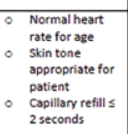 & 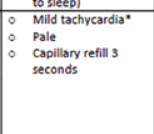 & 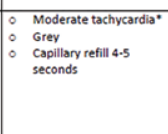 & 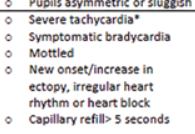 & \\
\hline Respiratory & $\begin{array}{l}\text { Normal } \\
\text { reppirator rate } \\
\text { for } \\
\text { for ofe } \\
\text { No retractions }\end{array}$ & 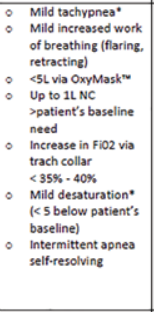 & 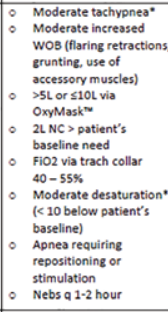 & 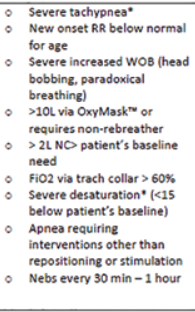 & \\
\hline $\begin{array}{l}\text { Staff concern: } \\
\text { add one point }\end{array}$ & (1) & (1) & (1) & (1) & \\
\hline $\begin{array}{l}\text { Family Concern: } \\
\text { add one point }\end{array}$ & (1) & (1) & (1) & (1) & \\
\hline
\end{tabular}

Follow guidelines for heart rate, respiratory rate, and oxygen saturations based on age and severity: normal, mild and moderate and severe.

Adapted from Boston Children's CHEWS
B

Activate RRT or Harvey Team at any time for immediate assistance

St. Jude Advanced Warning Score: Escalation of Care Algorithm

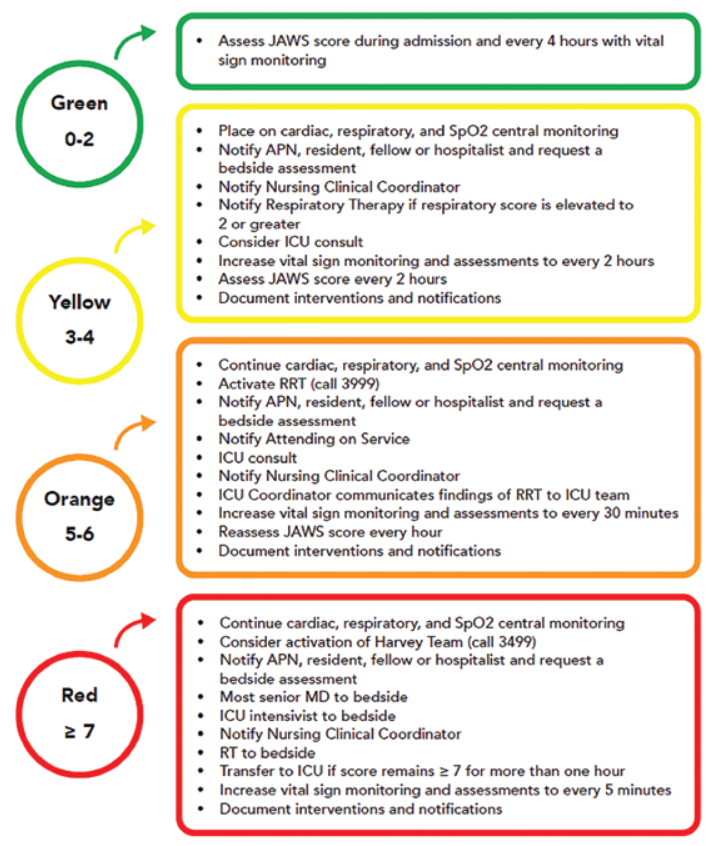

Patients with POST (DNAR) should not have care escalated based on their goals of care

Fig. 1. PEWS-St. Jude Advanced Warning System. The St. Jude Advanced Warning System scoring tool (A) and escalation algorithm (B) implemented at St. Jude Children's Hospital. The tool was adapted from the PEWS used at Boston Children's Hospital validated in pediatric oncology and hematopoietic stem cell transplant patients. ${ }^{1}$ Notes: RRT is the Rapid Response Team. Harvey Team is St. Jude's Code Blue Team.

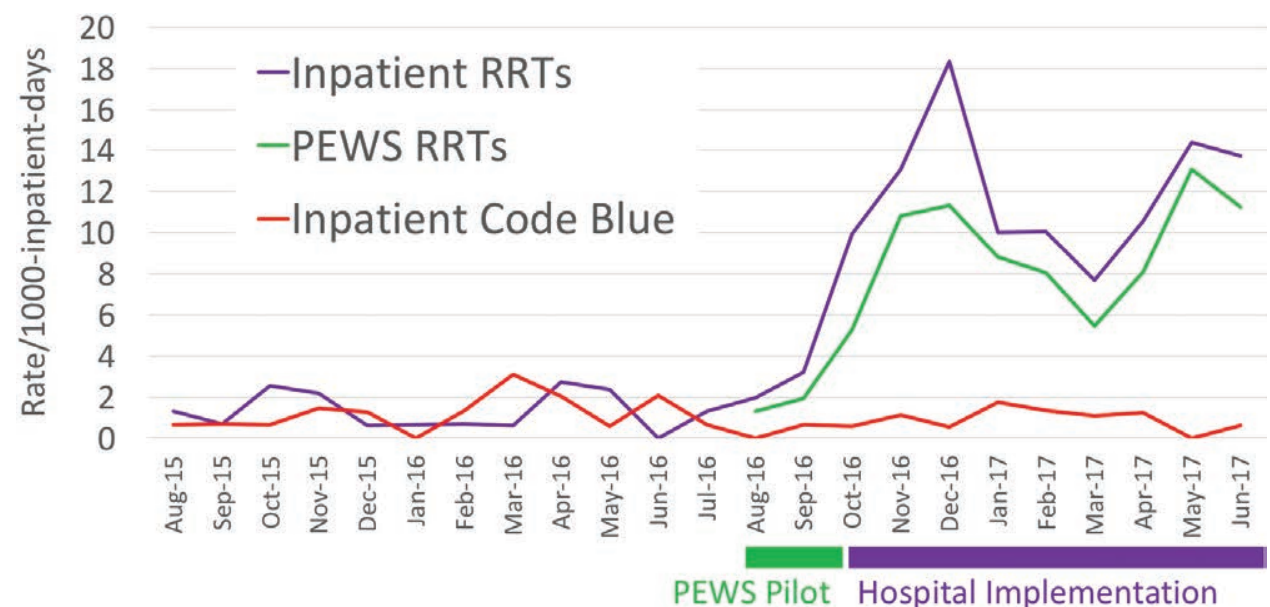

Fig. 2. Inpatient emergency activations before and after the implementation of a PEWS. Rate of total inpatient rapid response team (RRT) activations (purple), RRTs due to PEWS (green) and inpatient Code Blue (red) per 1,000-inpatient-days in the time before and after PEWS implementation. Frequency of RRT activations increased after PEWS implementation from 1.3 to 12.2/1,000-inpatient-days $(P<0.0001)$, without an increase in Code Blue activations $(1.2$ to $0.97 / 1,000$-inpatient-days, $P=0.49)$. 


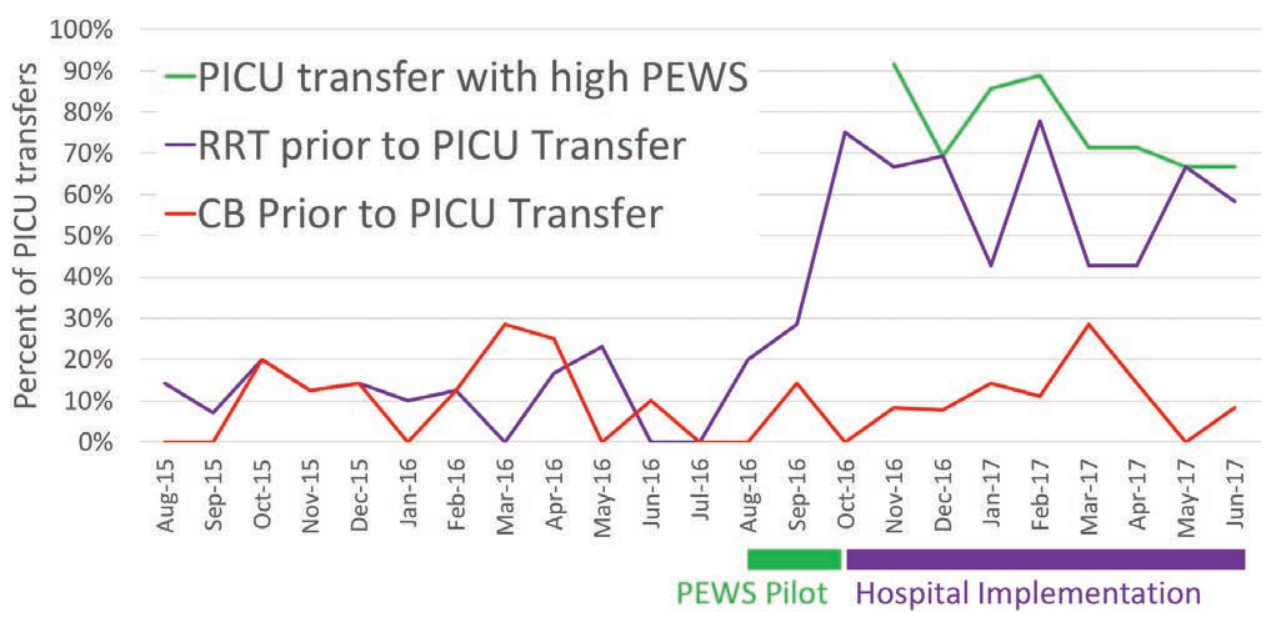

Fig. 3. Mechanism of unplanned PICU transfer before and after implementation of a PEWS. Percentage of unplanned PICU transfers with high PEWS in the 24 hours prior to PICU transfer (green), with RRT activation prior to PICU admission (purple) and with Code Blue activation prior to PICU admission (red) before and after PEWS implementation. Unplanned PICU transfers arriving via RRT increased from $11.5 \%$ to $60.5 \%$ of admissions $(P<0.0001)$. 